\title{
NONINVERTIBLE TRANSFORMATIONS ADMITTING NO ABSOLUTELY CONTINUOUS $\sigma$-FINITE INVARIANT MEASURE
}

\author{
JANE M. HAWKINS AND CESAR E. SILVA \\ (Communicated by R. Daniel Mauldin)
}

\begin{abstract}
We study a family of $n$-to- 1 conservative ergodic endomorphisms which we will show to admit no $\sigma$-finite absolutely continuous invariant measure. We exhibit recurrent measures for these transformations and study their ratio sets; the examples can be realized as $C^{\infty}$ endomorphisms of the 2-torus.
\end{abstract}

\section{INTRODUCTION}

In this paper we study a family of $n$-to- 1 conservative ergodic endomorphisms which we will show to admit no nontrivial $\sigma$-finite absolutely continuous invariant measure. Other examples in the literature that have been shown not to admit absolutely continuous finite invariant measures, e.g. [LY] and [GS], do admit infinite invariant measures. We also exhibit recurrent measures for our transformations and study their ratio sets; the existence of recurrent measures for any conservative ergodic endomorphism not admitting an invariant measure is an open problem [Si; ES]. Our examples can be realized as $C^{\infty}$ endomorphisms of the 2-torus; we do not know of conservative ergodic 1-dimensional continuous endomorphisms with no $\sigma$-finite absolutely continuous invariant measure.

Let $(X, \mathscr{B}, \mu)$ be a Lebesgue probability space. A nonsingular endomorphism $T$ is a measurable transformation on $X$ such that $\mu(N)=0$ if and only if $\mu\left(T^{-1} N\right)=0$. Since all the endomorphisms that we consider are finite-to-one we may assume (cf. [W]) that they are forward measurable and forward nonsingular. A Markovian function for $(T, \mu)$ is a positive finite a.e. measurable function $\omega$ such that, for all nonnegative measurable functions $f$,

$$
\int f \circ T \omega d \mu=\int f d \mu
$$

If $\omega$ is $T^{-1} \mathscr{B}$-measurable then this forces $\omega=\left(d \mu / d \mu T^{-1}\right) \circ T$ a.e. and we define it to be the Radon-Nikodym derivative of $T$ and denote it by $\omega^{\mu}$. For

Received by the editors November 2, 1989.

1980 Mathematics Subject Classification (1985 Revision). Primary 28D05, 58F11. 
$n \geq 0$ we define

$$
\omega(n, \cdot)=\omega \cdot \omega \circ T \cdots \omega \circ T^{n-1} .
$$

We note that $\omega(n, \cdot)$ is a Markovian function for $T^{n}$ but it need not be $T^{-n} \mathscr{B}$ measurable.

Define $\theta$ by

$$
\int f \circ T d \mu=\int f \theta d \mu, \text { for all } f \geq 0 .
$$

It follows that $\omega^{\mu}=1 /(\theta \circ T)$ a.e. We also recall the definition of Parry's Jacobian $J(x)$. By a theorem of Rohlin we can decompose the space into disjoint measurable subsets $A_{i}$ such that the restriction $T_{i}$ of $T$ to each $A_{i}$ is 1-to-1 from $A_{i}$ onto a.e. $X$, and for any measurable set $B$ in $A_{i}, \mu(B)=0$ implies $\mu\left(T_{i} B\right)=0$. Define $J_{i}=d \mu T_{i} / d \mu$ on $A_{i}$ and $J(x)=\sum \chi_{A_{i}} J_{i}(x)$. One can show that $\sum_{y \in T^{-1} x} 1 / J(y)=\theta(x)$ a.e.

All spaces are Lebesgue probability spaces. Equalities are mod 0 .

\section{EXAMPLES OF NONMEASURE PRESERVING ENDOMORPHISMS}

Let $T$ be a type III (cf. $\S 3)$ automorphism on $(X, \mathscr{B}, \mu)$ and $S$ a one-sided Bernoulli n-to- 1 measure preserving endomorphism acting on $(Y, \mathscr{C}, \nu)$. It is clear that $T \times S$ is a nonsingular $n$-to-1 endomorphism on $X \times Y$. Let $\omega=\omega^{\mu}$ denote the Radon-Nikodym derivative of $T$ with respect to $\mu$. If $\widehat{\omega}$ denotes the Radon-Nikodym derivative of $T \times S$ with respect to $\mu \times \nu$, since $\omega$ is $(T \times S)^{-1}(\mathscr{B} \times \mathscr{C})$ measurable it follows that $\widehat{\omega}(x, y)=\omega(x) \mu \times \nu$ a.e. We also have that $\widehat{\omega}(i, x, y)=\omega(i, x)$ a.e. We recall that $T$ is said to be $(\omega, \mu)$ recurrent if $\sum_{i=0}^{\infty} \omega(i, x, y)=\infty$ a.e.; if this holds for $\omega=\omega^{\mu}$ then we say that $T$ is $\mu$-recurrent or that the measure $\mu$ is recurrent. It has been shown in [HS] and [ST] that $T$ is $(\omega, \mu)$-recurrent if and only if $\omega$ is a recurrent cocycle.

Lemma 2.1. The endomorphism $T \times S$ is conservative and ergodic with respect to $\mu \times \nu$.

Proof. Since $T$ is conservative, $\sum_{i=0}^{\infty} \widehat{\omega}(i, x, y)=\infty$ a.e., and so [Si, Lemma 2], $T \times S$ is $\mu \times \nu$-recurrent and therefore by [Si, Lemma 3] $T \times S$ is conservative. Next we note that $S$ is an exact endomorphism, and since $T$ is ergodic, [ALW, Theorem 5.1] implies that $T \times S$ is ergodic.

Lemma 2.2. Let $R$ be a conservative ergodic nonsingular endomorphism on $(X, \mu)$. If $R$ has no equivalent invariant measure then $R$ has no nontrivial absolutely continuous $\sigma$-finite invariant measure.

Proof. Let $\nu$ be an $R$-invariant nontrivial measure absolutely continuous with respect to $\mu$. Suppose there is a set $N$ with $\nu(N)=0$ and $\mu(N)>0$. Let $N^{*}=\bigcup_{i=0}^{\infty} R^{-1} N$. Since $R$ is conservative $R^{-1} N^{*}=N^{*}(\bmod \mu)$. Ergodicity implies $\mu\left(N^{*} \Delta X\right)=0$; so $\nu\left(N^{*} \Delta X\right)=0$. But we know $\nu\left(N^{*}\right)=0$ so $\nu(X)=0$, a contradiction. 
Proposition 2.3 [ST]. Let $T$ be a nonsingular endomorphism with Markovian pair $(\omega, \mu)$. If $T$ is $(\omega, \mu)$-recurrent, then $T$ admits a $\sigma$-finite invariant measure equivalent to $\mu$ if and only if $\omega$ is a coboundary.

Theorem 2.4. Let $T$ be a type III automorphism and $S$ a one-sided Bernoulli endomorphism on $n$ symbols. Then $T \times S$ is an $n$-to-1 conservative ergodic nonsingular endomorphism of $(X \times Y, \mathscr{B} \times \mathscr{C}, \mu \times \nu)$ admitting no absolutely continuous $\sigma$-finite invariant measure.

Proof. By Lemmas 2.1, 2.2, and Proposition 2.3, it is enough to show that $\widehat{\omega}(x, y)=\omega(x)$ is not a $T \times S$ coboundary. We note that since $\widehat{\omega}(x, y)=$ $\omega(x), \sum_{i=0}^{\infty} \widehat{\omega}(i, x, y)=\infty$. Suppose there is a positive finite-valued measurable function $f$ on $X \times Y$ such that

$$
f(T x, S y) \omega(x)=f(x, y) \quad \mu \times \nu \text {-a.e. }
$$

Define $g(x, y, z)=f(x, y) / f(x, z)$. One computes that $g(T x, S y, S z)=$ $g(x, y, z)$, and since $T \times S \times S$ is ergodic (by the same argument as in Lemma 2.1) then $g$ is a constant $C$ a.e. That is, for $\mu$-a.e. fixed $x_{0} \in X$, almost all pairs $(y, z) \in Y \times Y$ satisfy $f\left(x_{0}, y\right)=C f\left(x_{0}, z\right)$. By reversing $y$ and $z$, we have for $\nu$-a.e. $z, f\left(x_{0}, z\right)=C f\left(x_{0}, y\right)=C^{2} f\left(x_{0}, z\right)$, so $C=0,-1$, or 1. By our hypothesis on $f$ we can eliminate the first two cases, so $C=1$. It follows that $f$ is a coboundary for $T$, a contradiction.

We now let $X=Y=S^{1}$, the unit circle, and $\mu=\nu=m=$ Lebesgue (Haar) measure on $S^{1}$. We define $f$ to be any ergodic type III $C^{\infty}$ diffeomorphism of $S^{1}$; such diffeomorphisms exist by [Ka]. Letting $g(z)=z^{n}$ on $S^{1}$, it follows from the theorem that $f \times g$ is a conservative ergodic $C^{\infty}$ endomorphism on the 2-dimensional torus admitting no $\sigma$-finite invariant measure absolutely continuous with respect to Haar measure, the smooth class for the manifold. The Jacobian of $f \times g$ is $J(f \times g)\left(z_{1}, z_{2}\right)=n f^{\prime}\left(z_{1}\right)$ where $f^{\prime}(z)$ is the derivative of $f$ at $z$. This implies that

$$
\theta_{1}\left(z_{1}, z_{2}\right)=1 / f^{\prime}\left(f^{-1} z_{1}\right)=\left(f^{-1}\right)^{\prime}\left(z_{1}\right) \text { and } \omega^{m \times m}\left(z_{1}, z_{2}\right)=f^{\prime}\left(z_{1}\right) \text {. }
$$

It has already been shown in [ES] that Proposition 2.3 is not true when $\omega$ is not recurrent, and it is an open problem whether every nonsingular conservative endomorphism admits an equivalent measure $\nu$ for which $\omega^{\nu}$ is recurrent. It was shown in [ES], however, that any $n$-to- 1 conservative ergodic endomorphism admits an equivalent (infinite $\sigma$-finite) nonrecurrent measure. From [ST] it follows that there always exist nonrecurrent Markovian pairs with respect to a finite equivalent measure; the Markovian function $\omega$ is not necessarily the Radon-Nikodym derivative in their construction. However we show below that one can always choose a finite measure with a nonrecurrent Radon-Nikodym derivative.

Proposition 2.6. Let $(X, \mathscr{B}, \mu)$ be a Lebesgue space, $m$ some infinite $\sigma$-finite measure equivalent to $\mu$, and $T$ a conservative ergodic n-to-1 nonsingular endomorphism on $X$. Then $m$ restricted to the $\sigma$-algebra $T^{-1} \mathscr{B}$ is $\sigma$-finite. 
Proof. It follows from the $n$-stack structure decomposition of [ES].

Corollary 2.7. Let $T$ be a conservc tive ergodic $n$-to-1 nonsingular endomorphism acting on a Lebesgue space $(X, \mathscr{B}, \mu)$. Then $T$ admits a finite measure $\nu$ equivalent to $\mu$ such that the Radon-Nikodym derivative of $T$ with respect to $\nu, \omega^{\nu}$, is nonrecurrent.

Proof. By Theorem 3.1 of [ES] $T$ has an equivalent measure $m$ such that $\omega^{m}$ is nonrecurrent. Since $m$ is $\sigma$-finite when restricted to $T^{-1} \mathscr{B}$ there exists a $T^{-1} \mathscr{B}$-measurable function $f$ such that $\int f d m=1$. We use $f$ as a transfer function: define the $\nu$ by $\nu=f m$. Since $f$ is $T^{-1} \mathscr{B}$-measurable it follows from [HS, Lemma 2.1] that $\omega^{m}$ and $\omega^{\nu}$ are cohomologous, therefore, $\omega^{\nu}$ must be nonrecurrent.

Question. Can one compute necessary or sufficient conditions for a nonrecurrent measure to have an equivalent $\sigma$-finite invariant measure?

We briefly show how to obtain finite-to-one nonconservative examples. Let $T$ be a conservative ergodic nonsingular automorphism such that $T^{2}$ is ergodic, and $S$ be defined on $\mathbf{N}$ by $S(n)=n-1$ if $n>1$, and $S(1)=2$. Then $T \times S$ is an ergodic endomorphism on the nonatomic space $X \times \mathbf{N}$ that is not conservative [Si]; clearly $T \times S$ cannot admit an equivalent finite invariant measure. If $T$ is type $\mathrm{II}_{1}$ then $T \times S$ has an infinite invariant measure, and if $T$ is type III then by inducing on the first two fibers one can see that $T \times S$ admits no equivalent $\sigma$-finite invariant measure.

\section{RATIO SETS}

There are various notions of a ratio set for an endomorphism which generalize the definition given by Krieger for automorphisms (cf. [HS] and [H]). Here we consider the ratio set as in [HS]. We denote the ratio set of $T$ with respect to $\mu$ by $r_{\mu}(T)$ and define it as follows.

$$
\begin{aligned}
r_{\mu}(T)= & \left\{\lambda \in \mathbf{R}^{+} \cup\{0\}: \text { for every } A \in \mathscr{B}, \mu(A)>0 \text { and every } \varepsilon>0\right. \text { there } \\
& \text { exists } \left.n \geq 1 \text { such that } \mu\left(A \cap T^{-n} A \cap\left\{x: \omega^{\mu}(n, x) \in N_{\varepsilon}(\lambda)\right\}\right)>0\right\},
\end{aligned}
$$

where $N_{\varepsilon}(1)$ is an $\varepsilon$-neighborhood of 1 in $\mathbf{R}, N_{\varepsilon}(\lambda)=\lambda N_{\varepsilon}(1)$ if $\lambda \neq 0$ and $N_{\varepsilon}(0)=\{t: t<\varepsilon\}$.

When $T$ is an automorphism, this is exactly the same as the ratio set defined by Krieger and is invariant under a change to any equivalent measure [Kri]. When $T$ is not invertible, the ratio set depends on the measure $\mu$ and not just on the measure class of $\mu$. This is because $1 \in r_{\mu}(T)$ if and only if $\omega^{\mu}$ is recurrent [HS].

The following properties of the ratio set have been shown to hold in both the invertible [Kri] and noninvertible case [HS].

(1) $r_{\mu}(T)$ is closed in $\mathbf{R}^{+} \cup\{0\}$. 
(2) $r_{\mu}(T) \cap \mathbf{R}^{+}$is a closed semigroup and is a group if and only if $\mu$ is recurrent.

(3) In the case that $r_{\mu}(T)$ is a group containing at least one element other than 1 we say that $T$ is type III.

If $r_{\mu}(T)=\mathbf{R}^{+} \cup\{0\}$ we say that $T$ is type III $_{1}$.

If $r_{\mu}(T)=\left\{\lambda^{n}: n \in \mathbf{Z}\right\} \cup\{0\}, 0<\lambda<1$, we say that $T$ is type III $_{\lambda}$.

If $r_{\mu}(T)=\{0,1\}$ we say that $T$ is type III $_{0}$.

It was shown in [HS] that if we start with a fixed measure $\mu$ and its associated Radon-Nikodym derivative $\omega^{\mu}$, then multiplying $\omega^{\mu}$ by a coboundary (a measurable function of the form $(h \circ T) / h$ for some positive measurable $h$ ) gives $\omega^{\nu}$ for $\nu=h \mu$ if and only if $h$ is $T^{-1} \mathscr{B}$-measurable. In this case we say $\nu$ is cohomologous to $\mu$, which is obviously stronger than just an equivalent measure. Otherwise the resulting product cocycle is still Markovian for $\nu \sim \mu$ and cohomologous to the pair $\left(\omega^{\mu}, \mu\right)$ but is not the Radon-Nikodym derivative for $\nu$ [ST].

Lemma 3.1. The ratio set of $T$ with respect to $\mu$ is invariant under a change to a cohomologous measure.

Proof. We assume that $\nu=h \mu$ and that $\omega^{\nu}=((h \circ T) / h) \omega^{\mu}$ by our hypothesis. For any $\lambda \in r_{\mu}(T)$, we show that $\lambda \in r_{\nu}(T)$; by symmetry of cohomologous measures this completes the proof. Now given any set $A \in \mathscr{B}, \mu(A)>0$, and any $\varepsilon>0$, we find a constant $c$ and a set $B \subset A$ such that $(1-.01 \varepsilon) c \leq$ $h(x) \leq(1+.01 \varepsilon) c$ for all $x \in B$. Then there exists $n \geq 1$ such that

$$
\mu\left(B \cap T^{-n} B \cap\left\{x: \omega^{\mu}(n, x) \in N_{.1 \varepsilon}(\lambda)\right\}\right)>0,
$$

and for every point in the above intersection we have that $\omega^{\nu}(n, x) \in N_{\varepsilon}(\lambda)$.

It follows from Proposition 2.3 and Lemma 3.1 that for recurrent measures the following result of Krieger generalizes to the noninvertible case (Proposition 3.5). As above, we assume $T$ is an ergodic and conservative endomorphism. We first recall the following variation of a well-known lemma (cf. [Kre] and [ST]).

Lemma 3.2. Let $T$ be a nonsingular endomorphism. Then $T$ admits an equivalent finite invariant measure if $\inf _{n} w(n, x)>0$.

Lemma 3.3. If there is some $\lambda \in r_{\mu}(T)$ with $\lambda \leq 1$ then $r_{\mu}(T)=\{1\}$ if and only if $0 \notin r_{\mu}(T)$.

Proof. Suppose that there is some $\lambda<1$ in $r_{\mu}(T)$. Then by [HS] $\lambda^{m} \in r_{\mu}(T)$ for every $m \geq 1$. Given any $A \in \mathscr{B}$ and any $\varepsilon>0$, we choose $m$ large enough so that $\lambda^{m}<\varepsilon / 3$. Then there exists an $n$ such that

$$
\begin{aligned}
& \mu\left(A \cap T^{-n} A \cap\left\{x: \omega^{\mu}(n, x) \in N_{\varepsilon / 3}\left(\lambda^{m}\right)\right\}\right)>0, \text { which implies that } \\
& \mu\left(A \cap T^{-n} A \cap\left\{x: \omega^{\mu}(n, x) \in N_{\varepsilon}(0)\right\}\right)>0, \text { since } \\
& N_{\varepsilon / 3}\left(\lambda^{m}\right) \subset(0, \varepsilon) .
\end{aligned}
$$


Therefore $0 \in r_{\mu}(T)$. The converse is trivial.

Corollary 3.4. Let $\mu$ be a recurrent measure. Then $r_{\mu}(T)=\{1\}$ if and only if there exists a set $A \in \mathscr{B}$ of positive measure and a constant $c>0$ such that $\omega_{A}(n, x)>c$ for all $n \geq 1$ and all $x \in A$ (where $\omega_{A}(1, x) \equiv \omega^{\mu}\left(n_{A}, x\right)$ ).

Proof. Since $0 \notin r_{\mu}(T)$ by Lemma 3.3 there exists a set $A \in \mathscr{B}, \mu(A)>0$, and $\varepsilon>0$, so that

$$
\mu\left(\bigcup_{n \geq 1} A \cap T^{-n} A \cap\left\{x: \omega^{\mu}(n, x) \in N_{\varepsilon / 3}(0)\right\}\right)=0 .
$$

This implies that $\omega_{A}(n, x)>\varepsilon$ for all $n$ and $x \in A$ (by removing a set of measure zero from $A$ if necessary).

The following proposition has been obtained independently and by a different proof by $\mathbf{P}$. Thieullen (unpublished).

Proposition 3.5. Let $\mu$ be a recurrent measure. Then $T$ admits an equivalent $\sigma$-finite invariant measure if and only if $r_{\mu}(T)=\{1\}$.

Proof. If $T$ has an equivalent invariant measure, then by Proposition $2.3 \omega^{\mu}$. is cohomologous to 1 . If $\nu$ is the invariant measure then clearly $r_{\nu}(T)=\{1\}$; by Lemma 3.1, $r_{\mu}(T)=\{1\}$.

Now suppose $r_{\mu}(T)=\{1\}$. From Corollary 3.4 there exists a set of positive measure $A$ and a constant $c$ such that uniformly on $A, \omega_{A}(n, x)>c$ for all $n \geq 1$. Then it follows that $\inf \omega_{A}(n, x)>0$. By Lemma 3.2 this implies that $T_{A}$ admits a finite invariant measure, and therefore $T$ admits a $\sigma$-finite invariant measure.

We use Proposition 3.5 to give another method for determining when many endomorphisms fail to admit invariant measures. We can easily compute the ratio set with respect to the product measure in our examples and show that it is the same as the ratio set of the automorphism $T$. Using the notation of Theorem 2.3 we prove the following.

Theorem 3.6. If $T$ is a type III $_{\lambda}$ automorphism, with $\lambda \in(0,1]$, then

$$
r_{\mu \times \nu}(T \times S)=r_{\mu}(T) \text {. }
$$

Proof. It is enough to show the result on rectangles by [ChHP]. Given $A \subset X$, $B \subset Y$, and $\varepsilon>0$, we first find $N$ large enough so that for all $n>N$, $\nu\left(B \cap S^{-n} B\right)>0$. We then find $n>N$ so that

$$
\mu\left(A \cap T^{-n} A \cap\left\{x: \omega^{\mu}(n, x) \in N_{\varepsilon}(\lambda)\right\}\right)>0 .
$$

It then follows that

$$
\mu \times \nu\left(A \times B \cap(T \times S)^{-n}(A \times B) \cap\left\{(x, y): \omega(n, x, y) \in N_{\varepsilon}(\lambda)\right\}\right)>0 .
$$


This shows that $r_{\mu}(T) \subset r_{\mu \times \nu}(T \times S)$. To show the reverse inclusion, we suppose that $\lambda \in r_{\mu \times \nu}(T \times S)$. Then given any rectangle $A \times B$, and $\varepsilon>0$, we can find an $n$ so that

$$
\mu \times \nu\left(A \times B \cap(T \times S)^{-n}(A \times B) \cap\left\{(x, y): \omega(n, x, y) \in N_{\varepsilon}(\lambda)\right\}\right)>0 .
$$

This means that $\mu\left(A \cap T^{-n} A \cap\left\{x: \omega^{\mu}(n, x) \in N_{\varepsilon}(\lambda)\right\}\right)>0$ by the definition of product measure and the fact that $\omega(n, x, y)=\omega^{\mu}(n, x)$.

Corollary 3.7. $r_{\mu}(T)=\{1\}$ if and only if $r_{\mu \times \nu}(T \times S)=\{1\}$.

Proof. By the proof of Theorem 3.6, $1 \in r_{\mu}(T)$ if and only if $1 \in r_{\mu \times \nu}(T \times S)$. If $r_{\mu}(T)=\{1\}$, then by Proposition 3.5, $r_{\mu \times \nu}(T \times S)=\{1\}$. The converse follows by Theorem 2.4 .

Corollary 3.8. $r_{\mu}(T)=\{0,1\}$ if and only if $r_{\mu \times \nu}(T \times S)=\{0,1\}$.

Proof. We first note that the proof of Theorem 3.6 cannot be used to show that $0 \in r_{\mu}(T)$ implies $0 \in r_{\mu \times \nu}(T \times S)$ (by arguments in [ChHP]). The forward implication follows from Theorem 2.4 and Proposition 3.5, however, the converse follows from the proof of Theorem 3.6.

Remark 3.9. Let $R^{*}$ denote the Maharam skew product of $R$, as defined for endomorphisms in [Si]. Let $T$ and $S$ be as in Theorem 2.4 and write $R=$ $T \times S$. Then it follows from above that $R^{*}$ is ergodic if and only if $R$ is type III ${ }_{1}$ (with respect to $\mu \times \nu$ ), generalizing to this case Schmidt's result for automorphisms [Sc, Corollary 5.4].

Remark 3.10. From the work of Krieger, we know that for invertible transformations of type III $\lambda$ for $\lambda \in(0,1]$ the ratio set provides a complete invariant up to orbit equivalence.

The situation is more complicated for endomorphisms. To begin with, it is not yet clear what the best notion of orbit equivalence is; there are several equivalences which have been studied in [HS] and [H]. This ratio set so far does not seem to be invariant under either, due to the fact that one might change from one measure to an equivalent one and change from a recurrent to a nonrecurrent measure. However, changing to an equivalent measure leaves one in the same isomorphism class (unless some additional restrictions are put on the isomorphism).

This is not the only problem that can arise. One can, for example, take two type III $\lambda_{\lambda}$ transformations $T$ and $T^{\prime}$ which are not isomorphic. Then

$$
r_{\mu \times \nu}(T \times S)=r_{\mu^{\prime} \times \nu}\left(T^{\prime} \times S\right)
$$

but we do not have isomorphism. Alternatively, one could change measures on the shift space to obtain $S$ and $S^{\prime}$, both measure preserving shifts with inequivalent measures. Again

$$
r_{\mu \times \nu}(T \times S)=r_{\mu \times \nu^{\prime}}\left(T \times S^{\prime}\right),
$$


but not only are they not isomorphic, they are not even equivalent in the weaker sense of associated equivalence relations [H]. What is true in our examples is that we obtain orbit equivalent invertible natural extensions of $T \times S$ and $T^{\prime} \times S^{\prime}$ if and only if the ratio sets of the two product transformations are the same, assuming the ratio sets contain some $\lambda \in(0,1)$.

\section{ACKNOWLEDGMENTS}

The authors would like to thank S. J. Eigen and V. S. Prasad for useful conversations concerning this paper. A special case of this example was constructed at a workshop on nonsingular transformations at Williams College, June 1989, in which they participated.

\section{REFERENCES}

[ALW] J. Aaronson, M. Lin, and B. Weiss, Mixing properties of Markov operators and ergodic transformations, and ergodicity of Cartesian products, Israel J. Math. 33 (1979), 198-224.

[ChHP] J. R. Choksi, J. M. Hawkins, and V. S. Prasad, Abelian cocycles for nonsingular ergodic transformations and the genericity of type III 1 transformations, Monat. Math. 103 (1987), 187-205.

[CoFW] A. Connes, J. Feldman, and B. Weiss, An amenable equivalence relation is generated by a single transformation, Ergodic Theory Dynamical Systems, 1 (1981), 431-450.

[ES] S. J. Eigen and C. E. Silva, $A$ structure theorem for $n$-to-1 endomorphisms and existence of nonrecurrent measures, J. London Math. Soc. (2) 40 (1989), 441-451.

[GS] P. Gora and B. Schmitt, Un exemple de transformation dilatante et $C^{1}$ par morceaux de l'intervale, sans probabilite absolument continue invariante, Ergodic Theory Dynamical Systems 9 (1989), 101-113.

[H] J. M. Hawkins, Ratio sets of endomorphisms which preserve a probability measure, Contemp. Math., vol. 94, 1989, pp. 159-169.

[HS] J. M. Hawkins and C. E. Silva, Remarks on recurrence and orbit equivalence of nonsingular endomorphisms (Dynamical Systems, Proc. Univ. Maryland), Lecture Notes in Math, vol. 1342, Springer-Verlag, 1988, pp. 281-290.

[Ka] Y. Katznelson, Sigma-finite invariant measure for smooth mappings of the circle, J. d'Analyse Math. 31 (1977), 1-18,

[Kre] U. Krengel, Ergodic theorems, de Gruyter, 1985.

[Kri] W. Krieger, On the Araki-Woods asymptotic ratio set and non singular transformations of a measure space, Lecture Notes in Math., vol. 160, Springer-Verlag, 1970, pp.158-177.

[LY] A. Lasota and J. A. Yorke, On the existence of invariant measures for piecewise monotonic transformations, Trans. Amer. Math. Soc. 186 (1973), 481-488.

[Sc] K. Schmidt, Lectures on cocyles of ergodic transformation groups, Macmillan, New Delhi, 1977.

[Si] C. E. Silva, On $\mu$-recurrent nonsingular endomorphisms, Israel J. Math. 61 (1988), 1-13. 
[ST] C. E. Silva and P. Thieullen, The subadditive ergodic theorem and recurrence properties of Markovian transformations, J. Math. Anal. Appl. (to appear).

[W] P. Walters, Roots of $n: 1$ measure preserving transformations, J. London Math Soc. 44 (1969), 7-14.

Department of Mathematics, University of North Carolina, Chapel Hill, North CarOLINA 27514

Department of Mathematics, Williams College, Williamstown, Massachusetts 01267 